\title{
TOPOLOGICAL RESTRICTIONS ON DOUBLE FIBRATIONS AND RADON TRANSFORMS
}

\author{
ERIC TODD QUINTO
}

\begin{abstract}
Given two manifolds $X$ and $Y$, the topological concept double fibration defines two integral Radon transforms $R: C_{0}^{\infty}(X) \rightarrow C^{\infty}(Y)$ and $R^{t}: C^{\infty}(Y) \rightarrow$ $C^{\infty}(X)$. For every $x \in X$ the double fibration specifies submanifolds of $Y, G_{x}$, all diffeomorphic to each other. For $g \in C^{\infty}(Y), x \in X$, the transform $R^{\prime} g(x)$ integrates $g$ over $G_{x}$ in a specified measure. Let $k$ be the codimension of $G_{x}$ in $Y$. Under the Bolker assumption, we show that $k=1,2,4$, or 8. Furthermore if $k=1$ then every $G_{x}$ is diffeomorphic to $S^{n-1}$ or $R P^{n-1}$, if $k=8$ then $G_{x}$ is homeomorphic to $S^{8}$. In the other cases $G_{x}$ is a cohomology projective space. This shows that the manifolds $G_{x}$ which occur are all similar to the $G_{x}$ for the classical Radon transforms.
\end{abstract}

1. Introduction. Radon transforms are important tools in group representations, scattering theory, and partial differential equations, as well as in computerized tomography [5], [9], [10], [12]. The goal of this article is to demonstrate some of the interplay between analysis-the Radon transform-and topology-the theory of fiber bundles.

We first informally discuss the Radon transform and our main result (Theorem 1), then we state the central definitions: the double fibration (Definition 1) and the Bolker assumption (4), and finally we state and prove Theorem 1.

Let $X$ and $Y$ be smooth manifolds of the same dimension. The Radon transform $R: C_{0}^{\infty}(X) \rightarrow C^{\infty}(Y)$ and its dual $R^{t}: C^{\infty}(Y) \rightarrow C^{\infty}(X)$ are defined using the topological concept of double fibration [2]. A double fibration for $X$ and $Y$ defines for each $y \in Y$, a submanifold of $X, H_{y}$, and, for each $x \in X$, a submanifold of $Y, G_{x}$, such that the submanifolds $H_{y}$ (respectively, $G_{x}$ ) are all diffeomorphic to each other. Specified measures on $Z, X$, and $Y$ define measures $\mu_{x}$ on each $G_{x}$ and $\mu_{y}$ on each $H_{y}$ [3]. Then for $f \in C_{0}^{\infty}(X)$ and $y \in Y$, the Radon transform $R f(y)$ is the integral of $f$ over $H_{y}$ in the measure $\mu_{y}$ and for $g \in C^{\infty}(Y)$ and $x \in X, R^{\prime} g(x)$ is the integral of $g$ over $G_{x}$ in the measure $\mu_{x}$ [3]. The Bolker assumption, a geometric condition on the double fibration, guarantees that $R^{t} R$ is invertible locally [3]. The other analytic properties of $R$ and $R^{t}$ are exciting in their own right and we refer to [3], [4], [10], [11] for more information.

An understanding of the topologies of the manifolds $G_{x}$ and $H_{y}$ is important for an understanding of the Radon transform. The main result of this article, Theorem 1 , will show the severe topological restrictions placed on the manifold $G_{x}$ by the

Received by the editors April 29, 1980.

1980 Mathematics Subject Classification. Primary 44A05; Secondary 55R25.

Key words and phrases. Generalized Radon transform, double fibration, Hopf invariant, projective space. 
Bolker assumption (4). If $k$ is the codimension of $G_{x}$ in $Y$, then $k$ must be either $1,2,4$, or 8. Also, if $k=1$, then $G_{x}$ is diffeomorphic to either $S^{n-1}$ or $R P^{n-1}$; if $k=8$ then $G_{x}$ is homeomorphic to $S^{8}$; in the other cases $G_{x}$ is a cohomology complex $(k=2)$ or quaternion $(k=4)$ projective space.

This shows that "essentially" the only manifolds $G_{x}$ that occur are: $S^{n-1}, R P^{n-1}, C P^{n / 2-1}, H P^{n / 4-1}$, and $S^{8}$; the $G_{x}$ for classical Radon transforms on hyperbolic spaces, Euclidean spaces, and projective spaces (see [4]).

Most of the topology in the proof of Theorem 1 was contributed by Michael Davis; the author is indebted to him for this. The author would also like to thank Mauricio Gutierrez, Ed Miller, and Franklin Peterson for their helpful suggestions, as well as Victor Guillemin for all of his guidance and help on this problem.

2. Definitions. To define the double fibration of Gel'fand et al. [2] we consider a submanifold $Z$ of $X \times Y$ that has certain properties. Assume the projections $\pi$ : $Z \rightarrow X$ and $\rho: Z \rightarrow Y$ are fiber maps and define, for $x \in X$ and $y \in Y, G_{x}=$ $\rho \pi^{-1}\{x\}$ and $H_{y}=\pi \rho^{-1}\{y\}$. We also assume that for each $x_{1}, x_{2} \in X$ and $y_{1}, y_{2} \in$ $Y$

$$
\begin{array}{ll}
G_{x_{1}}=G_{x_{2}} & \text { if and only if } x_{1}=x_{2}, \\
H_{y_{1}}=H_{y_{2}} & \text { if and only if } y_{1}=y_{2} .
\end{array}
$$

Definition 1. Let $X$ and $Y$ be connected, paracompact, orientable, smooth manifolds of dimension $n$ and let $Z$ be a closed, connected, oriented submanifold of $X \times Y$ of codimension $k, k>0$. Assume the projections $\pi: Z \rightarrow X$ and $\rho$ : $Z \rightarrow Y$ are fiber maps with connected fibers such that $\pi$ is proper and assume that (1) is satisfied then

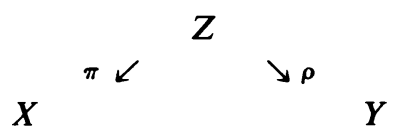

is a double fibration.

Definition 1 implies that each $H_{y}$ is a connected codimension $k$ submanifold of $X$ diffeomorphic to $\rho^{-1}\{y\}$ and each $G_{x}$ is a compact, connected, codimension $k$ submanifold of $Y$ diffeomorphic to $\pi^{-1}\{x\}$.

Given a double fibration (2), let $\Gamma$ denote $N^{*} Z-0$, the conormal bundle of $Z$ in $T^{*}(X \times Y)$ with its 0 section removed. Then $\Gamma$ is a closed, conic, Lagrangian submanifold of $T^{*}(X \times Y)-0$ [3]. Consider the projections

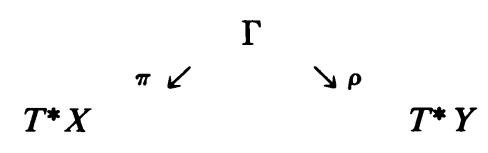

The Bolker assumption states that

$$
\rho: \Gamma \rightarrow T^{*} Y \text { is an injective immersion. }
$$

This is called the Bolker assumption because Ethan Bolker stated a similar assumption for finite Radon transforms. 
3. Main theorem. Throughout the article all homology and cohomology groups will have integer coefficients. We can now state our main theorem. This result is described in [3, p. 377].

THEOREM 1. Let (2) be a double fibration satisfying the Bolker assumption (4) and let $n$, the dimension of $X$, be greater than two. Let $k$ be the codimension of $Z$ in $X \times Y$. Then $k$ must be 1, 2, 4, or 8. Furthermore, for any $x \in X$

(i) if $k=1$ then $G_{x}$ is diffeomorphic to $S^{n-1}$ or $R P^{n-1}$;

(ii) if $k=2$ then $n$ is even and $G_{x}$ is homotopy equivalent to $C P^{n / 2-1}$;

(iii) if $k=4$ then four divides $n$ and $H^{*}\left(G_{x}\right)$ is isomorphic to the ring $H^{*}\left(H P^{n / 4-1}\right)$;

(iv) if $k=8$ then $n=16$ and $G_{x}$ is homeomorphic to $S^{8}$.

If $\rho$ is proper then the theorem holds for $H_{y}$.

Farshid Jamshidian [8] has shown that if (2) is a double fibration in the complex-analytic category with $X, Y$, and $Z$ compact and $k=2$, then the entire fibration (2) is diffeomorphic to the fibration for the classical Radon transform on $C P^{n}$ (see [4]).

Proof. Let $x_{0} \in X$ be fixed and let $G$ be the fiber $\pi^{-1}\left\{x_{0}\right\}$. Because $\pi$ is proper, $G$ is compact and, for any $x \in X, G$ is diffeomorphic to $G_{x}$. Let $E$ be the set of fibers of $\Gamma$ above $G$, that is $E=\left\{(x, \xi, y, \eta) \in \Gamma \mid x=x_{0}\right\}$. We have the following maps:

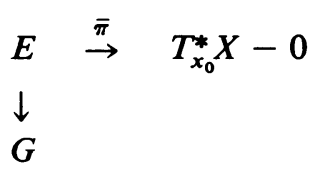

where $\bar{\pi}$ is $\pi$ restricted to $E$ and the vertical arrow is the projection to $G$. Because of the Bolker assumption, $\pi: \Gamma \rightarrow T^{*} X-0$ is a local diffeomorphism that is linear on cotangent coordinates [6]. This implies that $\bar{\pi}$ is a local diffeomorphism linear on the fibers of $E \rightarrow G$. Identify $S^{n-1}$ with the set of rays from the origin in $T_{x_{0}}^{*} X$ and let $S E$ be the sphere bundle of $E$. Define $f: S E \rightarrow S^{n-1}$ by the rule, for $\lambda \in$ $S E, f(\lambda)$ is the ray $\{t \bar{\pi}(\lambda) \mid t \geqslant 0\}$. Since $\bar{\pi}$ is a local diffeomorphism, $f$ is as well.

Assume that $S E$ is connected. Since $G$ is compact, the map $f$ is a local diffeomorphism from a compact connected set to $S^{n-1}$ for $n>2$ and hence $f$ is a diffeomorphism. Therefore, the map $S E \rightarrow G$ is a fibration with fiber $S^{k-1}(k=$ $\operatorname{codim} Z$ in $X \times Y$ ) and total space $S E=S^{n-1}$.

First, using the properties of the Hopf invariant [1], [14] we show that $k$ is either $1,2,4$, or 8 . Assume $k>1$; then, because $S E$ has connected base and connected fiber $S^{k-1}, S E$ is diffeomorphic to $S^{n-1}$. Applying the long exact homotopy sequence of the fibration shows that $G$ is $k-1$ connected. Therefore $S E$ is an oriented sphere bundle and the Gysin sequence with $\mathbf{Z}$ coefficients can be applied [7]. Let $\chi$ be the Euler class of the vector bundle $E, \chi \in H^{k}(G) \simeq H^{k}(E)$. Because $S E$ is diffeomorphic to $S^{n-1}$, the Gysin sequence of $G$ and $S E$ implies that

$$
H^{*}(G) \text { is a truncated polynomial algebra on one generator } \chi \in H^{k}(G) \text {. }
$$


Because $G$ is $k-1$ connected and $k>1$, the Hurewicz theorem implies that $\pi_{k}(G) \cong H_{k}(G) \cong \mathbf{Z}$. Let $g: S^{k} \rightarrow G$ be a generator of $\pi_{k}(G)$ and consider the pullback bundle $g^{*} S E$ :

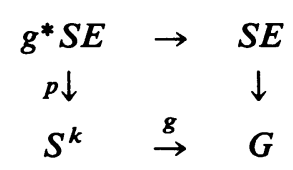

Since $\chi$ represents a generator of $H^{k}(G), g^{*} \chi$ is a generator of $H^{k}\left(S^{k}\right)$ as well as being the Euler class for the oriented sphere bundle $g^{*} S E$. Using a Gysin sequence again shows that $H^{*}\left(g^{*} S E\right) \cong H^{*}\left(S^{2 k-1}\right)$. Because $g^{*} S E$ is simply connected, the Whitehead theorem shows that $g^{*} S E$ is homotopy equivalent to $S^{2 k-1}$ by a map $r$.

We will now show that the map $h=p r: S^{2 k-1} \rightarrow g^{*} S E \rightarrow S^{k}$ has Hopf invariant one [14].

Let $D E$ be the disc bundle of $E$ and let $U \in H^{k}\left(g^{*} D E, g^{*} S E\right)$ be the Thom class (as in Theorem III 7.3 [7]). Because $g^{*} \chi$ generates $H^{k}\left(S^{k}\right)$, the Thom isomorphism theorem [7] implies that $p^{*} g^{*} \chi \cup U$ generates $H^{2 k}\left(g^{*} D E, g^{*} S E\right)$. By Proposition III 7.6 in [7] $U \cup U=p^{*} g^{*} \chi \cup U$. To summarize, $U$ is a generator of $H^{k}\left(g^{*} D E, g^{*} S E\right)$ and $U \cup U$ is a generator for $H^{2 k}\left(g^{*} D E, g^{*} S E\right)$.

Recall that $g^{*} S E$ is homotopic to $S^{2 k-1}$ and let $D_{a}$ denote the disc in $R^{2 k}$ of radius $a$. Let $T=S^{k} \cup_{h}\left(D_{1}-D_{1 / 2}\right)$ be the result of adjoining $D_{1}-D_{1 / 2}$ to $S^{k}$ via the map $h$ going from $S^{2 k-1}=\partial D_{1}$ to $S^{k}$. Because $g^{*} D E$ is contractible to $S^{k}$, the pair $\left(g^{*} D E, g^{*} S E\right)$ is homotopic to $\left(T, D_{3 / 4}-D_{1 / 2}\right)$. By excising $D_{1 / 2}$, $H^{*}\left(S^{k} \cup_{h} D_{1}, D_{3 / 4}\right)$ is naturally isomorphic to $H^{*}\left(T, D_{3 / 4}-D_{1 / 2}\right)$. Let $V \in$ $H^{k}\left(S^{k} \cup_{h} D_{1}, D_{3 / 4}\right)$ be the image of the Thom class $U \in H^{k}\left(g^{*} D E, g^{*} S E\right)$ under the equivalence above. Notice that $V$ generates $H^{k}\left(S^{k} \cup_{h} D_{1}, D_{3 / 4}\right)$ and $V \cup V$ generates $H^{2 k}\left(S^{k} \cup_{h} D_{1}, D_{3 / 4}\right)$.

Because $D_{3 / 4}$ is contractible, the long exact sequence of the pair shows that $H^{*}\left(S^{k} \cup_{h} D_{1}, D_{3 / 4}\right)$ is naturally isomorphic to $H^{*}\left(S^{k} \cup_{h} D_{1}\right)$ by the inclusion $j^{*}$. Therefore $j^{*} V$ generates $H^{k}\left(S^{k} \cup_{h} D_{1}\right)$ and $j^{*} V \cup j^{*} V$ generates $H^{2 k}\left(S^{k} \cup_{h} D_{1}\right)$. This shows that the map $h$ has Hopf invariant one and so, by the work of Adams [1], $k=1,2,4$, or 8 .

We now consider the cases (i)-(iv).

If $k=1$ then the fiber of $S E$ is two points, $S^{0}$. Assume $S E$ is connected, then, because $\bar{\pi}$ is a linear map on the fibers of $E, f$ maps each fiber of $S E$ to antipodal points of $S^{n-1}$. The following diagram gives an isomorphism between $G$ and $R P^{n-1}$; the vertical arrows are projections.

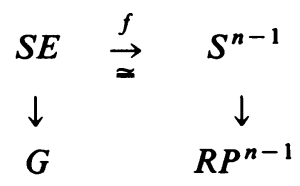

If $S E$ is disconnected then, because the map $f$ is a diffeomorphism on each component of $S E$, the set $S E$ is two copies of $S^{n-1}$ and $G$ is diffeomorphic to $S^{n-1}$. This proves (i).

Assume $k=2$; then (5) shows that $H^{*}(G)=H^{*}\left(C P^{n / 2-1}\right)$. In this case $S E \rightarrow G$ 
is an oriented circle bundle and so is equivalent to a principal circle bundle. The classifying map $G \rightarrow C P^{\infty}$ factors, after a homotopy, to a map $G \rightarrow C P^{n / 2-1}$. Since the map induces an isomorphism on $\pi_{1}$ and homology, $G$ is homotopy equivalent to $C P^{n / 2-1}$. This proves (ii).

If $k=4$ then the statement (5) proves (iii).

Finally assume $k=8$; then (5) implies that $H^{*}(G)$ is a truncated polynomial algebra on one generator in $H^{8}(G)$. By Theorem B in [1] the top dimension of $H^{*}(G)$ is either 8 or 16 . Assume the top dimension is 8 , then, because $G$ is simply connected and $H^{*}(G) \cong H^{*}\left(S^{8}\right), G$ is homotopy equivalent to $S^{8}$ by a generator of $\pi_{8}(G) \cong H_{8}(G)$. By the work of Smale [13] and others $G$ is actually homeomorphic to $S^{8}$. If $n=16$ then let $S$ be the Thom space of $G$ [7] and let $W \in H^{8}(S) \cong$ $H^{8}(D E, S E)$ be a generator. Then an argument using the Thom isomorphism theorem and Proposition III 7.6 of [7] shows that $W^{3}$ is a generator for $H^{24}(S)$. Using Theorem B of [1] on $S$ yields a contradiction and shows that this case cannot exist. This proves (iv) and the theorem.

\section{REFERENCES}

1. J. F. Adams and M. F. Atiyah, K-theory and the Hopf invariant, Quart. J. Math. Oxford Ser. (2) 17 (1966), 31-38.

2. I. M. Gel'fand, M. I. Graev and Z. Ya. Shapiro, Differential forms and integral geometry, Funkcional. Anal. i Priložen. 3 (1969), 24-40; English transl. in Functional Anal. Appl. 3 (1969), $101-114$.

3. V. Guillemin and S. Sternberg, Geometric asymptotics, Math. Surveys, Vol. 14, Amer. Math. Soc. Providence, R. I.,1977.

4. S. Helgason, The Radon transform on Euclidean spaces, compact two-point homogeneous spaces, and Grassmann manifolds, Acta Math. 113 (1965), 153-180.

5., A duality for symmetric spaces with applications to group representations, Advances in Math. 5 (1970), 1-154.

6. L. Hörmander, Fourier integral operators. I, Acta Math. 127 (1971), 79-183.

7. D. Husemoller, Fibre bundles, McGraw-Hill, New York, 1966.

8. F. Jamshidian, Integral geometry on plane complexes, Doctoral Dissertation, Harvard Univ., Cambridge, Mass., 1980.

9. P. D. Lax and R. S. Phillips, Scattering theory, Academic Press, New York, 1967.

10. D. Ludwig, The Radon transform on Euclidean space, Comm. Pure Appl. Math. 69 (1966), 49-81.

11. E. T. Quinto, The dependence of the generalized Radon transform on defining measures, Trans. Amer. Math. Soc. 257 (1980), 331-346.

12. K. T. Smith, D. C. Solmon and S. L. Wagner, Practical and mathematical aspects of the problem of reconstructing objects from radiographs, Bull. Amer. Math. Soc. 83 (1977), 1227-1270.

13. S. Smale, Generalized Poincaré conjecture in dimensions greater than four, Ann. of Math. (2) 74 (1961), 391-406.

14. N. E. Steenrod and D. B. A. Epstein, Cohomology operations, Princeton Univ. Press, Princeton, N. J., 1962.

Department of Mathematics, Tufts University, Medford, MassachusetTs 02155 